\title{
Detecting clinically actionable variants in the 3' exons of PMS2 via a reflex workflow based on equivalent hybrid capture of the gene and its pseudogene
}

Genevieve M Gould ${ }^{\dagger}$, Peter V Grauman ${ }^{\dagger}$, Mark R Theilmann ${ }^{\dagger}$, Lindsay Spurka, Irving E Wang, Laura M Melroy, Robert G Chin, Dustin H Hite, Clement S Chu, Jared R Maguire, Gregory J Hogan and Dale Muzzey* (B)

\begin{abstract}
Background: Hereditary cancer screening (HCS) for germline variants in the $3^{\prime}$ exons of PMS2, a mismatch repair gene implicated in Lynch syndrome, is technically challenging due to homology with its pseudogene PMS2CL. Sequences of PMS2 and PMS2CL are so similar that next-generation sequencing (NGS) of short fragments - common practice in multigene HCS panels-may identify the presence of a variant but fail to disambiguate whether its origin is the gene or the pseudogene. Molecular approaches utilizing longer DNA fragments, such as long-range PCR (LR-PCR), can definitively localize variants in PMS2, yet applying such testing to all samples can have logistical and economic drawbacks.
\end{abstract}

Methods: To address these drawbacks, we propose and characterize a reflex workflow for variant discovery in the $3^{\prime}$ exons of PMS2. We cataloged the natural variation in PMS2 and PMS2CL in 707 samples and designed hybrid-capture probes to enrich the gene and pseudogene with equal efficiency. For PMS2 exon 11, NGS reads were aligned, filtered using gene-specific variants, and subject to standard diploid variant calling. For PMS2 exons 12-15, the NGS reads were permissively aligned to PMS2, and variant calling was performed with the expectation of observing four alleles (i.e., tetraploid calling). In this reflex workflow, short-read NGS identifies potentially reportable variants that are then subject to disambiguation via LR-PCR-based testing.

Results: Applying short-read NGS screening to 299 HCS samples and cell lines demonstrated $>99 \%$ analytical sensitivity and $>99 \%$ analytical specificity for single-nucleotide variants (SNVs) and short insertions and deletions (indels), as well as $>96 \%$ analytical sensitivity and $>99 \%$ analytical specificity for copy-number variants. Importantly, $92 \%$ of samples had resolved genotypes from short-read NGS alone, with the remaining $8 \%$ requiring $L R-P C R$ reflex.

Conclusion: Our reflex workflow mitigates the challenges of screening in PMS2 and serves as a guide for clinical laboratories performing multigene HCS. To facilitate future exploration and testing of PMS2 variants, we share the raw and processed LR-PCR data from commercially available cell lines, as well as variant frequencies from a diverse patient cohort.

Keywords: PMS2, PMS2CL, Lynch syndrome, Hereditary cancer screening, Reflex testing

\footnotetext{
* Correspondence: research@counsyl.com

${ }^{\dagger}$ Genevieve M Gould, Peter V Grauman and Mark R Theilmann contributed

equally to this work.

Counsyl, 180 Kimball Way, South San Francisco, CA 94080, USA
}

(c) The Author(s). 2019 Open Access This article is distributed under the terms of the Creative Commons Attribution 4.0 International License (http://creativecommons.org/licenses/by/4.0/), which permits unrestricted use, distribution, and reproduction in any medium, provided you give appropriate credit to the original author(s) and the source, provide a link to the Creative Commons license, and indicate if changes were made. The Creative Commons Public Domain Dedication waiver (http://creativecommons.org/publicdomain/zero/1.0/) applies to the data made available in this article, unless otherwise stated. 


\section{Background}

Individual genomic variants inherited through the germline account for approximately $5 \%$ to $10 \%$ percent of cancer [1-3]. This heritable component can increase risk for malignancies across a range of tissues $[4,5]$ - such as breast, colorectal, pancreatic, and prostate-and is associated with pathogenic variants in $>100$ genes [6]. To assess patients' risk for such cancers, hereditary cancer screening (HCS) typically uses targeted next-generation sequencing (NGS) to detect relevant variants in the coding regions and select noncoding regions on a multigene testing panel.

In most genomic regions interrogated by HCS panels, NGS alone is sufficient to yield high sensitivity and specificity $[7,8]$; high accuracy is critical for HCS because test results prompt patients to alter their clinical-management decisions $[9,10]$. In a minority of regions, however, standard NGS strategies that use hybridization to capture and sequence short DNA fragments could incorrectly identify genotypes. Genes that pose particular challenges often have homologous sequences (e.g., pseudogenes) elsewhere in the genome that are captured and sequenced along with the gene itself, complicating alignment and the identification of variants specific to the gene.

PMS2 is commonly included on HCS panels due to its association with Lynch syndrome, though HCS panels often require orthogonal techniques to identify variants located in PMS2 [11-15]. Its nearby pseudogene, PMS2CL, complicates accurate NGS read alignment and variant identification in exons 11 through 15 at the 3' end of PMS2 (Fig. 1a): the coding sequences were previously reported to share $98 \%$ sequence identity with PMS2CL [16]. Further, sequence exchange and gene conversion between the two regions are sufficiently frequent that even the few non-identical bases in the reference genome (hg19) cannot be reliably attributed to the gene or pseudogene $[17,18]$. Long-range PCR (LR-PCR) using a gene-specific primer in exon 10 amplifies PMS2 specifically (Fig. 1b), and variants in the terminal five exons of PMS2 can then be identified via Sanger sequencing [19-21] or NGS [22] (Fig. 1c). Although identification of copy-number variants (CNVs) in PMS2 is possible from LR-PCR and Sanger sequencing, it is not straightforward, which has motivated parallel use of multiplex ligation-dependent probe amplification (MLPA) to detect large deletions and duplications [19-24].

Multiple testing strategies exist that can achieve high sensitivity and specificity in the last five exons of $P M S 2$ [18-20, 22, 25, 26]. Performing LR-PCR, MLPA, and hybrid-capture NGS on each screened sample was presented previously on a small cohort [22], but applying this combination to a large patient population would be resource intensive and complicate workflow logistics.

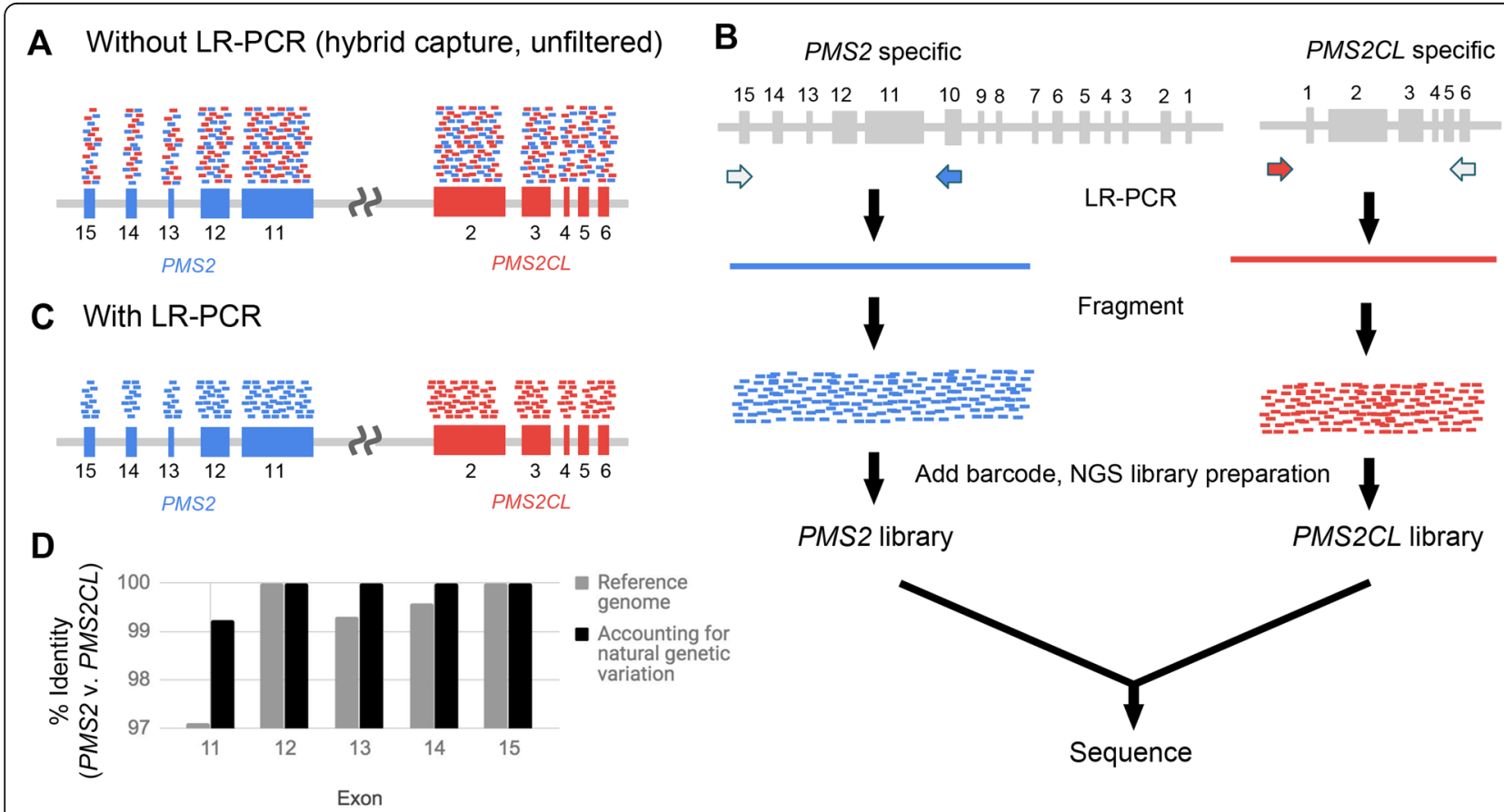

Fig. 1 LR-PCR strategy for building a dataset of natural genetic variation in PMS2 and PMS2CL. a Short-reads from NGS hybrid-capture data that originate from the gene (blue) and pseudogene (red) align to both the gene and pseudogene due to high homology. $\mathbf{b}, \mathbf{c}$ Using LR-PCR that is specific to the gene or pseudogene followed by fragmentation and barcoding (b), the resulting short NGS reads can be assigned to the gene or pseudogene (c). d Percent identity between the gene and pseudogene for PMS2 exons 11-15 based on the hg19 reference genome (gray) and after accounting for natural genetic variation obtained from LR-PCR samples (black) 
Herman et al. recently presented a method for identifying CNVs (but neither SNVs nor indels) in the terminal exons of $P M S 2$ or PMS2CL [26]. The method identified samples for follow-up LR-PCR testing to definitively localize the $\mathrm{CNV}$ to the gene or pseudogene. The authors noted a $\mathrm{CNV}$ false positive rate of $6.8 \%$, meaning that a significant portion of CNV-negative samples would unnecessarily undergo follow-up testing.

Here we present a reflex strategy for detection of SNVs, indels, and CNVs in the last five exons of PMS2. Our aim was to have the workflow's initial testing phase (i.e., upstream of reflex) be sensitive enough to maximize detection of PMS2 variants and sufficiently specific to minimize reflex burden. The proposed workflow applies hybrid-capture NGS to all samples and LR-PCR/MLPA only as a reflex assay. As the validity of LR-PCR in the last five exons of PMS2 is established [20, 21], we sought primarily to evaluate the performance of the hybrid-capture NGS assay via comparison to LR-PCR results from 299 clinical and cell line samples. We found that the workflow has high analytical accuracy while requiring reflex testing for only $8 \%$ of samples. Because our development of this workflow (schematized in Additional file 1: Figure S1) required collection of sequencing data and calculation of variant frequencies from a complicated genomic region with important impact on human health, we have made this information publicly available.

\section{Methods}

This study was reviewed and designated as exempt by Western Institutional Review Board and complied with the Health Insurance Portability and Accountability Act (HIPAA).

\section{Study samples}

Additional file 2: Table S1 indicates which sample sets were used for particular assays and analyses. Cell-line DNA was purchased from Coriell Cell Repositories (Camden, NJ) (Additional file 2: Table S2). Patient sample DNA was extracted from de-identified blood or saliva samples from patients who underwent Counsyl HCS testing. DNA samples with known positives were a gift from the Invitae Corporation.

\section{LR-PCR}

DNA was extracted and underwent an additional cleanup via incubation with $1 \times$ SPRI beads followed by $80 \%$ ethanol wash and elution into TE $(10 \mathrm{mM}$ Tris-HCl, 1 mM EDTA, pH 8.0). Approximately $300 \mathrm{ng}$ of eluted DNA served as the template in separate geneand pseudogene-specific LR-PCR reactions with the following final concentrations: $1 \times$ LongAmp Taq Reaction Buffer (New England Biolabs, NEB), 0.3 mM dNTPs, $1 \mu \mathrm{M}$ of a gene- or pseudogene-specific forward primer, $1 \mu \mathrm{M}$ of common reverse primer LRPCR_Unv_R (all primer sequences in Additional file 2: Table S3), 0.25\% Formamide, and 5 units LongAmp Hot Start Taq DNA Polymerase (NEB). Reactions including the gene-specific forward primer PMS2_LRPCR_F yielded a $\sim 17 \mathrm{~kb}$ amplicon spanning PMS2 exons 11-15 (the forward primer targets exon 10), whereas use of the pseudogene-specific forward primer PMS2CL_F amplified $\sim 18 \mathrm{~kb}$ from PMS2CL (spans region upstream of $P M S 2 C L$ through exon 6). Thermal-cycling involved initial denaturation at $94{ }^{\circ} \mathrm{C}$ for 5 min followed by 30 cycles of $94{ }^{\circ} \mathrm{C}$ for $30 \mathrm{~s}$ and $65^{\circ} \mathrm{C}$ for $18.5 \mathrm{~min}$. Final elongation was $18.5 \mathrm{~min}$ at $65{ }^{\circ} \mathrm{C}$, followed by a $4{ }^{\circ} \mathrm{C}$ hold. Quality of LR-PCR amplicons was assessed using $0.5 \%$ agarose gel electrophoresis and quantification with the broad range Qubit assay kit (Thermo Fisher).

Two different library-prep strategies were used to prepare LR-PCR amplicons for NGS. In the first, applied to patient samples, LR-PCR amplicons were fragmented by adding $2 \mu \mathrm{L}$ NEBNext dsDNA Fragmentase and NEBNext dsDNA Fragmentase Reaction Buffer v2 (1× final, $\mathrm{NEB})$ to the remaining LR-PCR reaction volume, and then incubated at $37^{\circ} \mathrm{C}$ for $25 \mathrm{~min}$. Addition of $100 \mathrm{mM}$ EDTA stopped the reaction, which underwent cleanup with $1.5 \times$ SPRI beads, followed by $80 \%$ ethanol wash and elution in TE. Fragmentation quality was assessed via Bioanalyzer (Agilent) with the High Sensitivity DNA kit. NGS library prep included end repair, A-tailing, and adapter ligation. Samples were PCR amplified with KAPA HiFi HotStart PCR Kit (Kapa Biosystems) for 8-10 cycles with barcoded primers with the following thermal cycling: initial denaturation at $95{ }^{\circ} \mathrm{C}$ for $5 \mathrm{~min}$ followed by cycles of $98{ }^{\circ} \mathrm{C}$ for $20 \mathrm{~s}, 60{ }^{\circ} \mathrm{C}$ for $30 \mathrm{~s}$, and $72{ }^{\circ} \mathrm{C}$ for $30 \mathrm{~s}$. The last elongation was $5 \mathrm{~min}$ at $72{ }^{\circ} \mathrm{C}$, followed by $4{ }^{\circ} \mathrm{C}$ hold. Library quality was verified via Bioanalyzer with a High Sensitivity DNA kit and the concentration was measured with absorbance via a microplate reader (Tecan Infinite M200 PRO).

The second approach to prepare LR-PCR amplicons for NGS-applied to the 155 cell-line samples-entailed fragmenting and inserting adapters into LR-PCR amplicons via tagmentation. Two duplex adapters were created by annealing single-stranded oligonucleotides: one duplex adapter had the Unv_Tn5_oligo (all primer sequences in Additional file 2: Table S3) annealed to Oligo A; the other duplex adapter had the Unv_Tn5_oligo annealed to Oligo B. The two separate annealing mixes included $25 \mu \mathrm{M}$ of each oligonucleotide in the duplex plus $1 \times$ annealing buffer $(10 \mathrm{mM}$ Tris- $\mathrm{HCl}, 50 \mathrm{mM} \mathrm{NaCl}, 1 \mathrm{mM}$ EDTA, $\mathrm{pH}$ 8.0). The reaction was denatured at $95{ }^{\circ} \mathrm{C}$ for $2 \mathrm{~min}$, incubated at $80{ }^{\circ} \mathrm{C}$ for $60 \mathrm{~min}$, stepped down in temperature by $1{ }^{\circ} \mathrm{C}$ every minute until reaching $20{ }^{\circ} \mathrm{C}$, and then held at $4{ }^{\circ} \mathrm{C}$. Adapters were loaded into the Tn5 enzyme during a $30 \mathrm{~min}$ incubation at $37{ }^{\circ} \mathrm{C}$ with 0.15 units of Robust Tn5 Transposase (kit from Creative Biogene), $1.25 \mu \mathrm{M}$ of each adapter, and $1 \times$ TPS buffer. 
LR-PCR amplicons were subjected to tagmentation with the Tn5-adapter construct. Tagmentation reactions occurred at $56{ }^{\circ} \mathrm{C}$ for $10 \mathrm{~min}$ in $1 \times \mathrm{LM}$ Buffer, with $0.5 \mu \mathrm{L}$ of loaded Tn5 and 1-2 ng of DNA from each LR-PCR reaction. After incubating, SDS (0.02\% final) was added to each reaction and incubated for $5 \mathrm{~min}$ to dissociate Tn5 from the DNA. Tagmention cleanup with $1 \times$ SPRI beads preceded molecular barcoding and amplification via PCR to generate NGS libraries. The PCR reaction included 1 unit Kapa HiFi Polymerase (Kapa Biosystems), 1× HiFi Buffer, $375 \mu \mathrm{M}$ dNTPs, $0.5 \mu \mathrm{M}$ of each primer, and the cleaned-up tagmented sample. Cycling started with gap-filling at $72{ }^{\circ} \mathrm{C}$ for $3 \mathrm{~min}$ and followed with 10 cycles of denaturation at $98{ }^{\circ} \mathrm{C}$ for $30 \mathrm{~s}$, annealing at $63{ }^{\circ} \mathrm{C}$ for $30 \mathrm{~s}$, and extension at $72{ }^{\circ} \mathrm{C}$ for $3 \mathrm{~min}$. Cleanup of NGS libraries was performed with $1 \times$ SPRI beads.

For patient samples, LR-PCR libraries were sequenced on a HiSeq 2500 (Illumina) in rapid run mode (paired reads, 150 cycles each). For cell line samples, LR-PCR libraries were sequenced on a NextSeq 550 (Illumina) to a minimum depth of 500 reads (single read, 150 cycles).

\section{Hybrid capture and sequencing}

Targeted NGS was performed as described previously [7, 8]. Briefly, DNA from a patient's blood or saliva sample was isolated, quantified by a dye-based fluorescence assay, and then fragmented to 200-1000 bp by sonication. Fragmented DNA was converted to an NGS library by end repair, A-tailing, and adapter ligation. Samples were then amplified by PCR with barcoded primers, multiplexed, and subjected to hybrid capture-based enrichment with 40-mer oligonucleotides (Integrated DNA Technologies) complementary to regions common between PMS2 and PMS2CL. NGS was performed on a HiSeq 2500 with mean sequencing depth of $\sim 500 \times$ for the whole panel (coverage in PMS2 is $\sim 1000 \times$ ). All target nucleotides are required to be covered with a minimum depth of 20 reads.

\section{Read alignment}

For hybrid-capture data, in order to aggregate PMS2and PMS2CL-originating reads at the PMS2 locus in the reference genome, paired-end NGS reads were first aligned to the hg19 human reference genome using BWA-MEM (version 0.17) [27]. The alignment at PMS2 exon 11 was filtered to only include reads that overlapped with a site of known difference between gene and pseudogene. Reads that aligned to PMS2 exons 12-15 and reads that aligned to PMS2CL exons 3-6 were partitioned into a BAM file using samtools [28]. The BAM file was converted into two unaligned FASTQ files (each member of the read pair parsed to one of the two files) using Picard (Broad Institute). Each single-end FASTQ file was separately realigned to the hg19 genome using BWA-MEM allowing for ambiguous alignments. In order to ensure each read aligns to both PMS2 and $P M S 2 C L$, the BWA-MEM parameters were set to the following values for 115 bp reads: "-a" to emit all alignments; seed length of 11 ; gap open penalty of 2 ; mismatch penalty of 1 ; and an alignment score threshold of 20. The resulting single-end alignments were used to generate a paired-end alignment in the following manner: 1) both single-end reads had the same read name; 2) both single-end reads mapped to the region spanning PMS2 exons $12-15$; 3) both single-end reads aligned within $1000 \mathrm{bp}$ of each other, and 4) when multiple putative pairs met the above conditions for a given read name, the pair with the highest alignment score was chosen. Reads that could not form proper pairs as described above were discarded. The resulting paired-end BAM file contained reads originating from both PMS2 and PMS2CL mapped to the PMS2 sequence.

For RT-PCR data (described below) and LR-PCR data, NGS reads were aligned to the hg19 genome sequence in which the PMS2CL sequence was removed, thereby aggregating genic and pseudogenic reads in PMS2.

\section{SNV and Indel calling}

For the PMS2 region into which reads from PMS2 and PMS2CL were mapped (see above), SNVs and short indels were identified using GATK 4.0 HaplotypeCaller [29] with the sample-ploidy option set to four, the max-reads-per-alignment-start option off, and the min-pruning option set to one. For the diploid PMS2 exon 11 region, SNVs and short indels were identified using GATK 1.6 [30] and FreeBayes [31]. For diploid SNV calling in the LR-PCR data, GATK 1.6 was similarly used. For the LR-PCR sample in which we suspected allelic dropout (see Discussion), AB was determined by visual inspection of the NGS data in the Integrative Genomics Viewer [32].

\section{CNV calling}

For short-read NGS data of hybrid-captured fragments, CNVs in PMS2 exon 11 were determined by measuring the relative NGS read depth at target positions using the algorithm described previously [7]. To call CNVs in PMS2 exons 12-15 from BAM files in which PMS2- and $P M S 2 C L$-originating reads were positioned in the $P M S 2$ sequence (see "Read Alignment" above), two modifications to the CNV calling algorithm were made: 1 ) the expected wild type copy number was changed from two to four copies, and 2) $p_{C N V}$ the parameter determining how likely the HMM is to transition from a wild-type to a CNV state, was set to 0.01 to obtain high CNV sensitivity and specificity from empirical data.

For CNV calling from LR-PCR data, read depth was counted in equal-sized bins (50 bp) that tile the amplicon. Bin counts for each sample were normalized by the 
median bin depth of the sample; next, each bin's values were normalized by the median of the bin. The same bins were used for corresponding regions of PMS2 and PMS2CL. The resulting binned and normalized data were searched for CNVs using the algorithm described previously [7]. CNV no-calls were manually reviewed to resolve status as positive or negative.

\section{CNV simulations}

Single-copy duplications and deletions were introduced by modifying the number of observed reads in one of the CNV-negative samples in a given batch of samples, as described previously [33]. For PMS2 exons 12-15, where baseline copy-number was four, single-copy deletions and duplications were introduced by subsampling reads to $75 \%$ or scaling read number by $125 \%$, respectively. Simulated CNVs were created for every possible contiguous combination of exons in the last 4 exons in PMS2. For each CNV size and position, 2186 samples were simulated and tested via the CNV calling algorithm, and sensitivity was calculated as the percentage of the synthetic CNVs that were correctly detected. CNVs were simulated separately in PMS2 exon 11, which had a baseline copy number of two, because pseudogenic reads were filtered from the genic sequence.

\section{Tetraploid Indel simulations}

Indels in a tetraploid background (relevant for exons 12-15 of PMS2, where gene- and pseudogene-originating reads were remapped) were simulated to better test indel-calling sensitivity using GATK4. Two diploid alignments, at least one of which was previously determined via HCS testingto contain an indel, were merged to create a tetraploid alignment. If one of the samples had more reads than the other in the $100 \mathrm{bp}$ region centered on the indel, reads were binomially downsampled such that each merged diploid sample had approximately the same number of aligned reads. Indels were then called from these synthetic tetraploid alignments using GATK4 as described in section SNV and Indel Calling above.

\section{Variant Curation}

For all variants in the last five exons of $P M S 2$, variant interpretation was performed in accordance with American College of Medical Genetics and Genomics (ACMG) criteria using a 5 tier classification category system (Benign, Likely Benign, Variant of Uncertain Significance, Likely Pathogenic, Pathogenic) [34]. Classifications were made using evidence available in the published literature and publicly available databases. Allele-frequency based rules were not used because of potentially inaccurate PMS2 variant identification in population databases. Variant classifications were reviewed and approved by board-certified laboratory directors.

\section{MLPA}

MLPA was performed according to manufacturer's protocol (MRC Holland, probemix P008-C1 PMS2 protocol issued 12/11/17 and MLPA General Protocol issued on 3/23/18). Generally, genomic DNA was covered with mineral oil to reduce evaporation during hybridization and ligation; next, DNA was denatured for 5 min at $98{ }^{\circ} \mathrm{C}$ and then held at $25^{\circ} \mathrm{C}$. Hybridization reagents and probemix were added to the samples and incubated at $95{ }^{\circ} \mathrm{C}$ for $1 \mathrm{~min}$ followed by $16-20 \mathrm{~h}$ at $60^{\circ} \mathrm{C}$. Probe pairs that bind target DNA at adjacent positions were ligated for $15 \mathrm{~min}$ at $54{ }^{\circ} \mathrm{C}$ and then amplified via PCR for 35 cycles. Amplified probes were mixed with ROX ladder and formamide and then separated on a capillary electrophoresis instrument. Coffalyser software (MRC Holland) normalized PMS2 probe intensities to those of the reference probes first within each sample and then among samples. Normalized probe intensities of each sample were compared to the average intensities of the reference samples; Coffalyser emitted CNV calls in the region.

\section{Reflex rate estimate}

The reflex rate was estimated using SNV-, indel-, and CNV-specific reflex rates from the LR-PCR and hybrid-capture data and subsequently extrapolating to a large cohort size using Markov Chain Monte Carlo simulations with pymc [35].

\section{Distinguishing Base analysis}

NGS reads from LR-PCR amplicons from PMS2 and $P M S 2 C L$ were aligned to $P M S 2$, and variants were called with GATK UniversalGenotyper as described in section SNV and Indel Calling above. Sites were considered reliable if variants were homozygous for the reference allele in the PMS2-specific amplicon and homozygous for an alternate allele in the $P M S 2 C L$-specific amplicon (as aligned to $P M S 2$ ) in $100 \%$ of samples.

\section{RNA testing RNA extraction and reverse transcription}

RNA was extracted from 33 samples with the Agencourt RNAdvance Blood kit (Beckman Coulter) from $400 \mu \mathrm{L}$ of whole blood following the manufacturer's instructions. RNA was extracted from blood tubes no more than seven days after blood draw was performed. Extraction quality was assessed with the RNA 6000 Nano kit (Agilent). RNA was quantified with Qubit HS RNA Assay kit (Thermo Fisher).

RNA was reverse transcribed using Superscript II Reverse Transcriptase with oligo-dT and random hexamers as primers (kit from Thermo Fisher). Reactions were performed as follows: $0.1-1.0 \mu \mathrm{g}$ total RNA, $1.25 \mu \mathrm{M}$ of both random hexamers and oligo-dT primer, $0.8 \mathrm{mM}$ 
dNTPs, and water up to a final volume of $12 \mu \mathrm{L}$. Reactions were heated at $65{ }^{\circ} \mathrm{C}$ for $5 \mathrm{~min}$ and then chilled on ice for $5 \mathrm{~min}$. $1 \times$ first-strand buffer and $0.01 \mathrm{M}$ DTT were added to each reaction and incubated at $42{ }^{\circ} \mathrm{C}$ for 2 min. $10 \mathrm{U} / \mu \mathrm{L}$ Superscript II Reverse Transcriptase was added to each reaction and incubated at $42{ }^{\circ} \mathrm{C}$ for $50 \mathrm{~min}$, then heat inactivated at $72{ }^{\circ} \mathrm{C}$ for $15 \mathrm{~min}$. A positive control of pooled mRNA (Stratagene, Catalog \#750500-41) was used with each reverse transcription reaction.

Following reverse transcription, RNA was hydrolyzed with $2 \mu \mathrm{L} 1 \mathrm{~N} \mathrm{NaOH}$ and heated at $95{ }^{\circ} \mathrm{C}$ for $5 \mathrm{~min}$. $4 \mu \mathrm{L}$ of $1 \mathrm{M}$ Tris-HCL pH 7.5 was used to neutralize the reaction for downstream processing. Qubit ssDNA Assay kit (Thermo Fisher) was used to quantify cDNA.

\section{$P C R$}

For each sample, two reactions were set up: 1) forward primer PMS2_RNA_F and reverse primer RNA_Unv_R amplified $1.5 \mathrm{~kb}$ of $P M S 2$ from $\mathrm{cDNA}$ and 2) forward primer PMS2CL_F and reverse primer RNA_Unv_R amplified $1.5 \mathrm{~kb}$ of $P M S 2 C L$ from cDNA (primer sequences in Additional file 2: Table S3). PCR reactions contained 1× LongAmp Taq Reaction Buffer (NEB), $0.3 \mathrm{mM}$ dNTPs, $1 \mu \mathrm{M}$ of each forward and reverse primer, 20-70 ng cDNA, $0.1 \mathrm{U} / \mu \mathrm{L}$ LongAmp Taq DNA polymerase (NEB), and water up to $25 \mu \mathrm{L}$. Thermocycling was as follows: $94{ }^{\circ} \mathrm{C}$ for $5 \mathrm{~min}, 30$ cycles of $94{ }^{\circ} \mathrm{C}$ for $30 \mathrm{~s}$, annealing at $52{ }^{\circ} \mathrm{C}$ for PMS2 and $55{ }^{\circ} \mathrm{C}$ for $P M S 2 C L, 65^{\circ} \mathrm{C}$ for $2 \mathrm{~min}$, followed by a final extension at $65{ }^{\circ} \mathrm{C}$ for $10 \mathrm{~min}$ and then a $4{ }^{\circ} \mathrm{C}$ hold. PCR products were cleaned with $1.2 \times$ SPRI beads. Amplicons were visualized with a $2 \%$ agarose gel or with the DNA 7500 kit (Agilent).

\section{Sequencing}

$50-100 \mathrm{ng}$ of each amplicon were fragmented in $50 \mu \mathrm{L}$ volumes with a Bioruptor (Diagenode) for 12 cycles, $30 \mathrm{~s}$ on and $90 \mathrm{~s}$ off. Fragmentation was visualized with High Sensitivity DNA kit (Agilent). All fragmented material was used as input for library preparation. KAPA Hyper Prep kit (Kapa Biosystems) was used for library preparation, and manufacturer instructions were followed. Adapters were diluted to $15 \mu \mathrm{M}$ for PMS2 and $3 \mu \mathrm{M}$ for PMS2CL. Nine cycles of enrichment PCR were performed. Samples were quantified using absorbance measurements (Tecan M200), normalized to $10 \mathrm{nM}$, and consolidated into one reaction. The final library was quantified with qPCR using KAPA Library Quantification Kit (Kapa Biosystems) and sequenced on the NextSeq 550 System (Illumina) for 75 cycles single read with dual indexing.

\section{Alignment}

Basecall files were converted to FASTQ files using bcl2fastq (Illumina). FASTQ files were aligned using STAR [36].

\section{Analytical metrics}

Metrics were defined as follows: Sensitivity $=\mathrm{TP} /(\mathrm{TP}+\mathrm{FN})$; Specificity $=\mathrm{TN} /(\mathrm{TN}+\mathrm{FP})$. The CIs were calculated by the method of Clopper and Pearson [37]. For SNVs and indels, true negatives were defined as concordant negative results observed at sites found to be polymorphic in our cohort (positions at which we observed non-reference bases in at least one sample).

\section{Results}

Zero nucleotides can reliably distinguish exons $12-15$ of PMS2 from PMS2CL

NGS of short DNA fragments would only be able to identify PMS2-specific variants in the last five exons if the fragments themselves could be unambiguously aligned to the gene or pseudogene. To overcome pseudogene interference, unique mapping would rely on the bases that differ between $P M S 2$ and PMS2CL. In the hg19 reference genome, these distinguishing bases are scarce (Fig. 1d, light bars): sequence identity in each of the last five exons of PMS2 (padded with $20 \mathrm{nt}$ of intronic sequence) exceeds $97 \%$, and the differences comprise only $26,0,1,1$, and 0 bases in exons 11 through 15 , respectively. Further, previous reports noted that natural variation may suppress the reliability of these distinguishing bases represented in the reference genome $[17,18]$.

To test the reliability of the reference genome, we assembled a catalog of natural variation in PMS2 exons 11-15 and the corresponding regions in PMS2CL. We performed NGS on gene- and pseudogene-specific LR-PCR amplicons on 707 of the patient samples in our cohort (Table 1) with diverse self-reported ethnicities (Additional file 3: Table S4). We found that 7 of the 26 expected positions in PMS2 exon 11 had distinct alleles in the gene and pseudogene, making them reliable distinguishing bases that enabled unique mapping of paired-end reads. In contrast, for 19 positions in exon 11 and two positions in exons 12-15, the ostensibly PMS2-specific alleles from hg19 were observed at least once in the PMS2CL LR-PCR data, and vice versa (see Additional file 3: Table S4 for allele frequencies). Therefore, after accounting for the natural variation in gene and pseudogene, there are zero reliable distinguishing bases (i.e., $100 \%$ sequence identity) in PMS2 exons $12-15$, and seven distinguishing bases in exon 11 (Fig. 1d, dark bars). Together, these data suggest that variant identification via short-read NGS alone could be sufficient for exon 11, but a different approach is required for exons 12-15.

\section{Reflex workflow to disambiguate variants discovered with short-read NGS}

We evaluated the plausibility of a workflow for the 3' exons of PMS2 that uses short-read NGS as its foundation and performs reflex testing with orthogonal assays to disambiguate the genic or pseudogenic origin of variants only when clinically needed (Fig. 2a). In the short-read NGS 
Table 1 Summary of samples

\begin{tabular}{ll}
\hline Assay & Samples \\
\hline LR-PCR + NGS & 718 patient samples \\
& 155 cell-line samples \\
Hybrid capture + NGS & 144 patient samples \\
& 155 cell-line samples \\
& 3 known positives \\
MLPA & 4 patient samples \\
& 4 cell-line samples \\
RT-PCR + NGS & 33 samples \\
\hline
\end{tabular}

stage of testing, the molecular approach is consistent across the last five exons of PMS2: DNA fragments are captured in a manner that is agnostic to their genic or pseudogenic origin by designing capture probes that specifically avoid positions shown to vary between PMS2 and PMS2CL in our LR-PCR data from patient samples (Fig. 2b, purple box).

The workflow employs different bioinformatics strategies for PMS2 exon 11 and for the group of exons 12-15 (Fig. 2b, blue box). For exon 11, we identified PMS2-specific variants by tailoring the read-alignment software to partition reads to PMS2 or PMS2CL based on the geneand pseudogene-distinguishing bases. By contrast, for
PMS2 exons 12-15, reads are aligned with permissive settings such that each read will align to both its best genic location and its best pseudogenic location (see Methods). For the typical sample with two copies each of PMS2 and $P M S 2 C L$, this approach effectively provides read depth in each location corresponding to four copies. To identify $\mathrm{SNVs}$, indels, and CNVs, we adjust the variant calling software such that it anticipates a baseline ploidy of two in exon 11 and four in exons 12-15 (Fig. 2b, blue and green boxes).

Disambiguation via reflex testing is only required for a subset of variants based on their type and clinical interpretation (Fig. 2b, orange box). As such, variant interpretation is performed prior to reflex testing. Benign variants are not reflex tested nor reported to patients. Samples with CNVs in any of the last five exons of PMS2 that are classified as pathogenic, likely pathogenic, or variants of uncertain significance (VUS) undergo reflex testing for disambiguation. Samples with non-benign SNVs or indels in exons $12-15$ are reflex tested for disambiguation, but samples with such variants in exon 11 are simply reported without reflex due to unique read mapping in that exon. Disambiguation testing for SNVs, indels, and CNVs can be performed via LR-PCR followed by sequencing to determine if the variant came from PMS2 or PMS2CL; MLPA can assist resolution of CNVs [20].
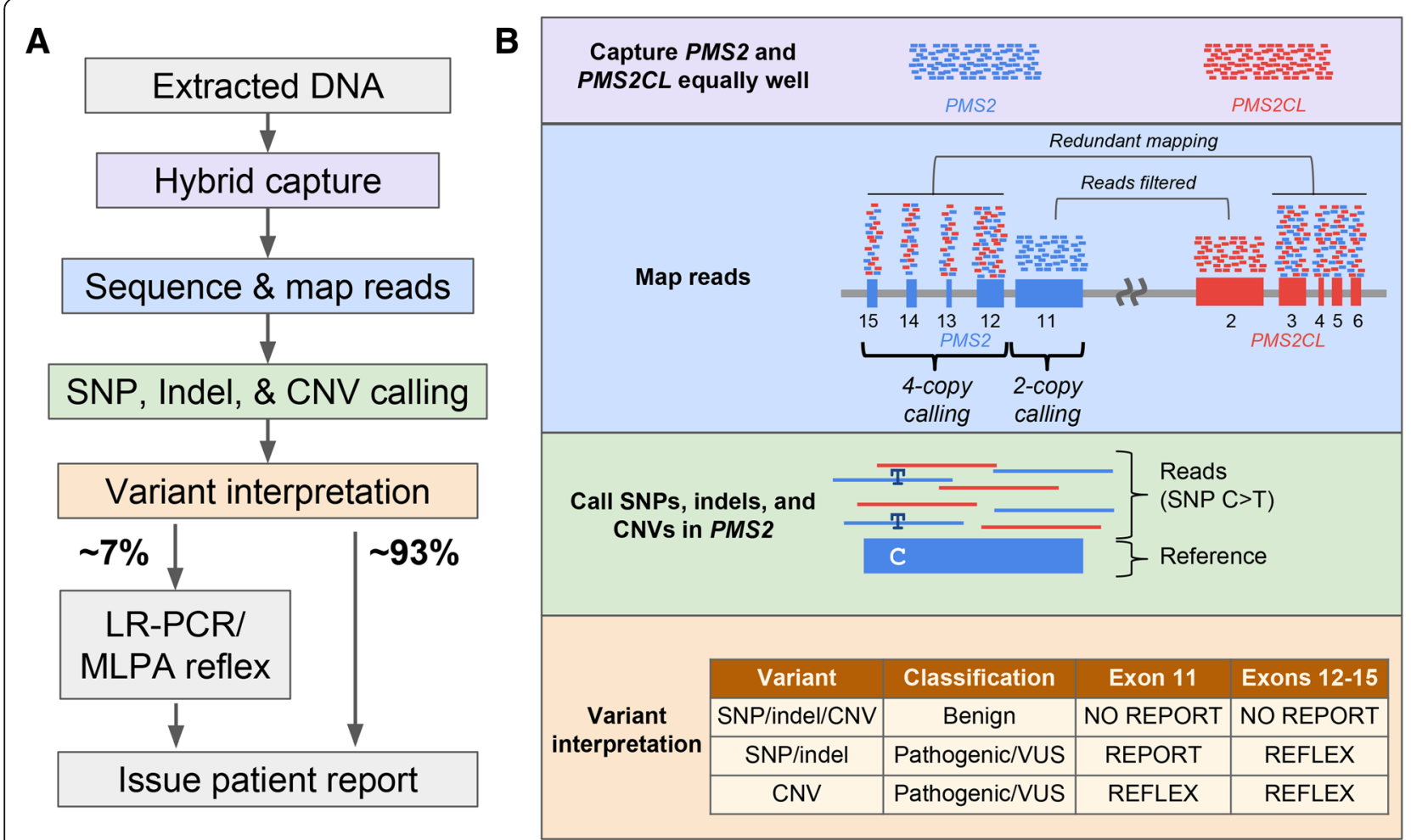

Fig. 2 Reflex workflow for variant identification in the last exons of PMS2. a Overview of sequencing and analysis workflow for the last five exons of PMS2. Colored nodes correspond to boxes in (b). b Details corresponding to workflow steps in (a); the details of each box are described in Methods and Results. "No report" means the variant does not appear on patient reports. "Reflex" means the sample is sent for LR-PCR-based disambiguation to determine if the variant is localized to the gene or pseudogene 
Executing the proposed workflow resolves cancer risk associated with the last five exons of PMS2 for the majority of samples with short-read NGS alone. For each of the 707 patient samples that underwent LR-PCR (Table 1), we performed variant classification on the results and found that nearly $93 \%$ could forgo reflex testing. The remaining $\sim 7 \%$ would have required subsequent testing to yield confident PMS2 screening results (Fig. 2a). The SNV- and indel-specific component of this reflex rate was 41/707 (5.8\%), and the reflex rates due to CNV calls and no-calls were $2 / 707(0.3 \%)$ and $1 / 144$ (0.7\%), respectively. Using simulations (see Methods), we estimated the reflex rate on a larger cohort of 13,000 patients to be $7.7 \%$ (95\% CI: 5.4$10.7 \%)$. We expect the $0.7 \%$ contribution to the reflex rate from samples with $\mathrm{CNV}$ no-calls to be an upper-bound estimate because our standard practice of retesting such samples at least once on short-read NGS typically yields a confident negative call (data not shown), thereby avoiding reflex testing. Therefore, we anticipate the overall reflex rate of the proposed workflow would be less than $8 \%$.

\section{Short-read NGS accurately identified samples needing reflex testing for SNVs and indels}

Our proposed reflex workflow is only clinically viable if the short-read NGS test (Fig. 2b) has high analytical sensitivity and specificity for (1) identifying variants in PMS2 exon 11 and (2) flagging samples that need reflex testing for variants in exons 12-15 with ambiguous PMS2/PMS2CL origin. To evaluate accuracy of the short-read NGS testing for SNVs and indels, we compared its results to those observed with LR-PCR for 144 patient samples and 155 cell lines (Fig. 3). Measuring genotype concordance in exons $12-15$ required an atypical confusion matrix because short-read NGS genotypes were reported as tetraploid (see Methods), whereas the LR-PCR returned diploid genotype calls for both the

\begin{tabular}{|l|c|c|c|c|c|}
\hline \multirow{2}{*}{ Concordance Type } & \multicolumn{4}{c|}{ LR-PCR } & \multicolumn{2}{c|}{ Hybrid Capture } \\
\cline { 2 - 7 } & PMS2 & PMS2CL & $\begin{array}{c}\text { Counts } \\
P M S 2 \text { alt, } \\
P M S 2 C L \text { alt }\end{array}$ & $\begin{array}{c}P M S 2+ \\
P M S 2 C L\end{array}$ & $\begin{array}{c}\text { Counts } \\
{[\# \text { alt alleles] }}\end{array}$ \\
\hline i. True positive & A/A & A/T & 0,1 & A/A/A/T & 1 \\
\hline ii. Permissible Dosage Error & A/A & T/T & 0,2 & A/A/A/T & 1 \\
\hline iii. False Positive & A/A & A/A & 0,0 & A/A/A/T & 1 \\
\hline iv. False Negative & A/A & A/T & 0,1 & A/A/A/A & 0 \\
\hline
\end{tabular}

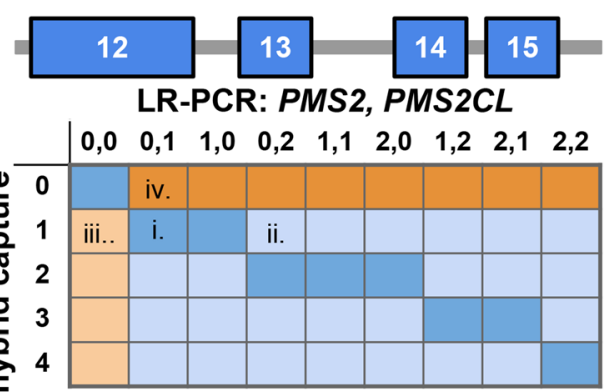

B

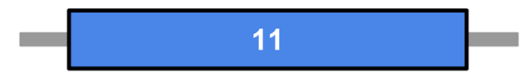

LR-PCR: PMS2

\begin{tabular}{|c|c|c|c|}
\hline & \multicolumn{3}{|c|}{ LR-PCR: PMS2 } \\
\hline & 0 & 1 & 2 \\
\hline \multirow{3}{*}{ 흘 } & 5003 & 0 & 0 \\
\hline & 0 & 387 & 0 \\
\hline & 0 & 0 & 291 \\
\hline
\end{tabular}

SNV Sensitivity: 100\% [99.5-100\%] SNV Specificity: 100\% [99.9-100\%] Indel Sensitivity: 100\% [2.5-100\%] Indel Specificity: 100\% [98.8-100\%]
C

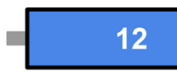

13

LR-PCR: PMS2, PMS2CL

\begin{tabular}{|c|c|c|c|c|c|c|c|c|c|}
\hline & 0,0 & 0,1 & 1,0 & 0,2 & 1,1 & 2,0 & 1,2 & 2,1 & 2,2 \\
\hline \multirow{5}{*}{ 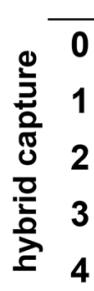 } & 6029 & 1 & 0 & 0 & 0 & 0 & 0 & 0 & 0 \\
\hline & 0 & 362 & 347 & 0 & 0 & 0 & 0 & 0 & 0 \\
\hline & 0 & 0 & 0 & 202 & 102 & 53 & 0 & 0 & 0 \\
\hline & 0 & 0 & 0 & 0 & 0 & 0 & 59 & 15 & 0 \\
\hline & 0 & 0 & 0 & 0 & 0 & 0 & 0 & 0 & 6 \\
\hline
\end{tabular}

SNV Sensitivity: 99.9\% [99.5-100\%] (0\% dosage errors) SNV Specificity: $100 \%$ [99.9-100\%] Indel Sensitivity: 100\% [80.5-100\%] (0\% dosage errors) Indel Specificity: 100\% [99.8-100\%]

Fig. 3 Hybrid-capture and LR-PCR are concordant for SNVs and indels. a Hypothetical examples to describe the concordance table for comparison of hybrid capture and LR-PCR data. All examples assume the reference base is A and the alternate ("alt") base is T. (i) Example of a true positive (dark blue) where an alt allele is present in PMS2CL. (ii) Example of a permissible dosage error (light blue), where PMS2CL is homozygous for the alt allele but hybrid capture only calls one alt allele instead of two. (iii) Example of a false positive (light orange), where only hybrid capture detected an alt allele. (iv) Example of a false negative (dark orange), where an alt allele in PMS2CL was missed by hybrid capture. Shaded matrix on the right indicates cells that represent true positives, permissible dosage errors, false positives, and false negatives. Numbers on axes denote the total number of alt alleles in either the hybrid capture data or the PMS2/PMS2CL LR-PCR data. b Diploid SNV and indel concordance for exon 11 of PMS2. Numbers on axes denote the number of alt alleles where 0 is equivalent to $0 / 0,1$ is equivalent to $0 / 1$, and 2 is equivalent to 1/1. 95\% confidence intervals in brackets. $\mathbf{c}$ Fourcopy SNV and indel concordance for exons 12-15 of PMS2/PMS2CL, as explained in (a) 
gene and pseudogene (Fig. 3a highlights several examples). The matrix includes "Permissible Dosage Errors," where the presence of alternate alleles is properly detected but the number of alternate alleles is discordant; such errors are deemed permissible because the presence of alternate alleles in short-read NGS would suffice to trigger reflex testing and be corrected. When compared at 1678 sites with LR-PCR as a truth set, short-read NGS testing had $100 \%$ analytical sensitivity and $100 \%$ analytical specificity in exon 11 (Fig. 3b), and 99.9\% analytical sensitivity and $100 \%$ analytical specificity in exons 12-15 (Fig. 3c).

The scarcity of indel calls in our patient cohort and cell lines (17 overall)-coupled with the uncommon usage of variant-calling software in a tetraploid-background mode for a clinical genomics application-motivated a deeper examination of indel-calling efficacy in PMS2 exons 12-15. We simulated the expected NGS data for samples with a tetraploid genome background populated with indels of different allele dosages (1, 2, 3, or 4 copies). To construct such samples, we merged the diploid NGS data from two samples (at least one containing an indel) in a region of our HCS test other than PMS2 (Fig. 4a, see Methods). The respective genotypes of the two samples provided an expected genotype of the merged sample: for instance, combining a heterozygous sample (one indel allele) with a homozygous-alternate sample (two indel alleles) would give an expected indel dosage of three. Figure $4 \mathrm{~b}$ illustrates
99.6\% sensitivity for indels in the simulated tetraploid background, suggesting that sensitivity is comparably high in exons 12-15 in PMS2 where our read-alignment and variant-calling strategy yields a tetraploid background. Because the empirical data in Fig. 3c demonstrate 100\% specificity for indels in exons 12-15, we did not further evaluate specificity with our simulations.

In sum, our comparison of SNV and indel calls between LR-PCR and short-read NGS suggests the pre-reflex step of our proposed workflow achieves sufficient analytical sensitivity and specificity to be considered for clinical use.

\section{Accurate detection with short-read NGS of samples needing CNV reflex testing}

To evaluate the sensitivity and specificity of short-read NGS for CNVs in the last five exons of PMS2, we tested patient samples, cell lines, known positives, and samples with simulated positives. As with SNVs and indels, we adapted our CNV detection algorithm to use a copy-number baseline of two for PMS2 exon 11 and four for exons 12-15 (Fig. 2b, blue box; see Methods). The three known-positive samples with CNVs in the last five exons were correctly identified as harboring CNVs encompassing the expected exons (Fig. 5a). We additionally observed a deletion of exon 13-14 in four of the cell lines and one of our clinical samples; for the clinical sample, short-read NGS identified a drop in signal from the tetraploid background (Fig. 5b), MLPA confirmed the

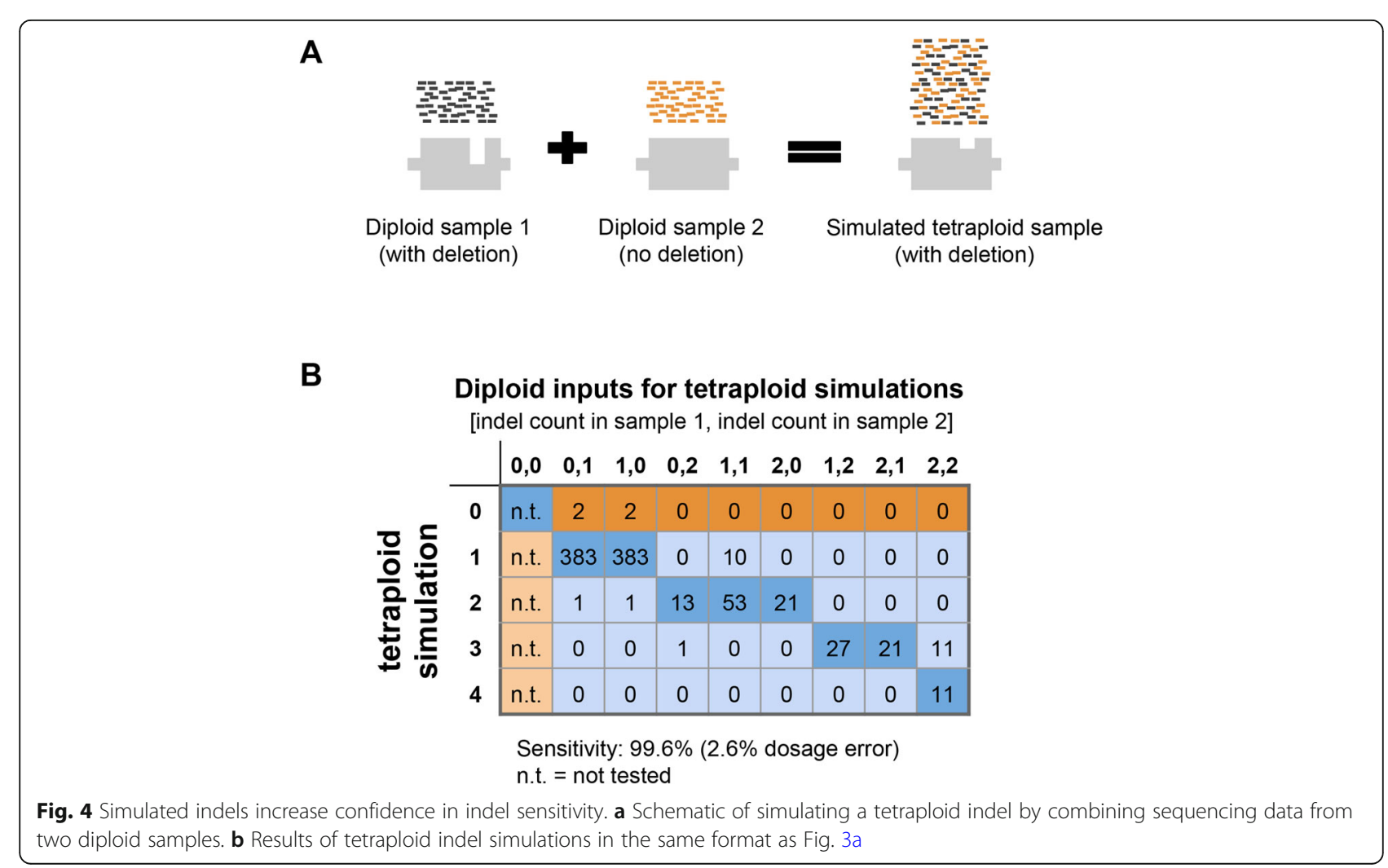




\begin{tabular}{|c|c|c|c|c|c|}
\hline & & & & & \\
\hline \multirow{2}{*}{ Sample } & \multicolumn{2}{|c|}{ PMS2 Hybrid Capture Call } & \multicolumn{2}{|c|}{\begin{tabular}{c|} 
Genotype \\
(LR-PCR or Known)
\end{tabular}} & \multirow{2}{*}{$\begin{array}{c}\text { Additional } \\
\text { Confirmation }\end{array}$} \\
\hline & Exons & Type & PMS2 & PMS2CL & \\
\hline Known Positive & $11-12$ & duplication & duplication & WT & none \\
\hline Known Positive & 12 & deletion & deletion & WT & none \\
\hline \begin{tabular}{|l|} 
Patient \\
\end{tabular} & $13-14$ & deletion & WT & deletion & MLPA \\
\hline Patient & $13-14$ & no call & WT & WT & MLPA \\
\hline HG02721 & $13-14$ & deletion & WT & deletion & MLPA \\
\hline HG02769 & $13-14$ & deletion & WT & deletion & MLPA \\
\hline HG02852 & $13-14$ & deletion & WT & deletion & MLPA \\
\hline HG03452 & $13-14$ & deletion & WT & deletion & MLPA \\
\hline Known Positive & 14 & deletion & deletion & WT & none \\
\hline
\end{tabular}

\section{B Hybrid capture}

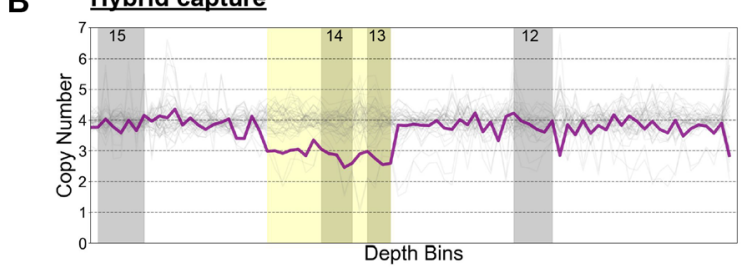

C MLPA

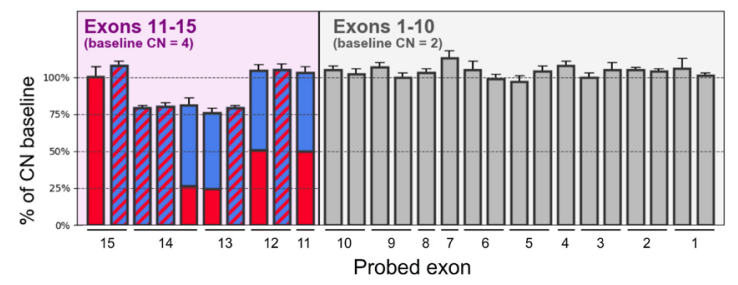

D $\quad$ LR-PCR

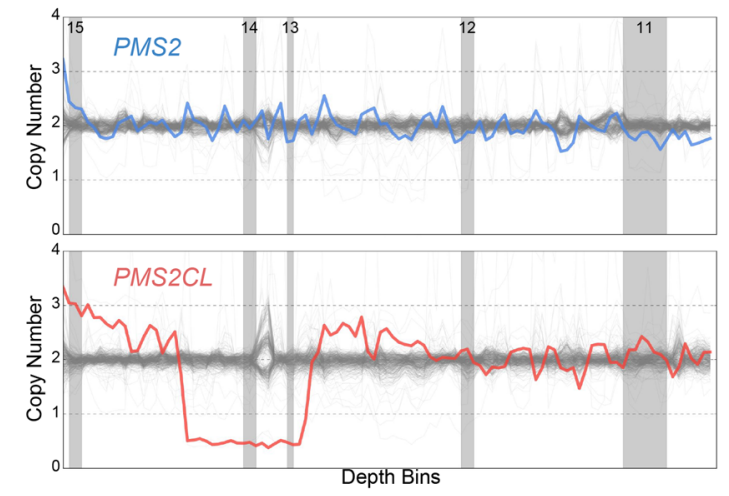

Fig. 5 Hybrid capture, LR-PCR, and MLPA are concordant for CNVs. a All CNVs called in the hybrid capture data and corresponding orthogonal confirmation data. b Hybrid capture data for the patient sample with an exon 13-14 deletion depicts copy-number estimates across the locus (bins). Gray regions denote the last four exons of PMS2. White regions denote introns. Yellow box indicates region of the CNV call. c MLPA data for the exon 13-14 deletion patient sample. PMS2-specific (solid blue), PMS2CL-specific (solid red), and PMS2/ PMS2CL degenerate MLPA probes (blue and red stripes) show the deletion in exons 13-14 of PMS2CL. d LR-PCR data for the exon 13-14 deletion sample depicting copy number estimates across the locus (bins) for PMS2 (blue, top) and PMS2CL (red, bottom). Gray regions depict exons 11-15 of PMS2 and white regions depict introns as in (b) presence of a similar deletion (Fig. 5c), and NGS on the LR-PCR amplicons revealed that the deletion was in PMS2CL rather than PMS2 (Fig. 5d). Interestingly, though only one of two copies of this region is deleted in PMS2CL, the LR-PCR profile shows a 75\% signal drop in the deleted region. We speculate that this arises from preferential amplification of the shorter deletion-harboring allele during LR-PCR. Therefore, although the LR-PCR data were unique in providing disambiguation, the short-read NGS and MLPA data had more readily interpretable copy-number values.

Due to the absence of a large catalog of CNV-positive samples, thorough and direct characterization of PMS2 CNV calling sensitivity with short-read NGS would require blind testing of thousands of samples. Instead, we used sequencing data from the abundance of $\mathrm{CNV}$-negative patients as substrate in simulations that introduce CNVs of given length and location (see Methods). By running our $\mathrm{CNV}$ detection algorithm on the 2186 simulated samples, we measured the analytical sensitivity for CNVs ranging from one to five exons in length (Table 2; simulation data on cell-line samples in Additional file 2: Table S6). Sensitivity for multi-exon deletions generally exceeded $99.2 \%$ and for single-exon deletions was $\sim 89 \%$. Weighing the simulated sensitivities by the observed frequency distribution of $\mathrm{CNV}$ length in the last five exons of PMS2 [21, 23, 24], we estimate that aggregate CNV sensitivity in this complicated genomic region is $96.7 \%$.

High sensitivity for CNVs must not come at the expense of low specificity, prompting us to measure the $\mathrm{CNV}$ false-positive rate in our large cohort. In our hybrid capture cohort of 302 samples, there was one no-call, which we treat as a false positive. Therefore, sample-level specificity is $99.7 \%$ (95\% CI: $98.2-100 \%$ ).

Based on these analyses, we conclude that short-read NGS-as optimized in our described workflow-can achieve $>96 \%$ sensitivity and $>99 \%$ specificity for detecting samples with CNVs in the terminal five exons of PMS2.

\section{Gene- and pseudogene-specific variant information for common cell lines}

Reference cell lines with known genotypes facilitate development and validation of novel molecular diagnostic methods, yet samples with high-quality genotypes in the PMS2 region are generally unavailable due to the region's complicated nature. In the course of developing and testing the workflow characterized above, we performed NGS of both hybrid-capture fragments and LR-PCR amplicons on cell lines where high-quality genome assemblies were publicly available from whole-genome sequencing with $\sim 30 \times$ depth (Illumina Polaris 1 diversity panel) or from the genome in a bottle (GIAB) consortium 
Table 2 CNV simulations demonstrate high analytical sensitivity

\begin{tabular}{|c|c|c|c|c|c|c|c|c|c|c|c|c|}
\hline \multirow[b]{2}{*}{ Size (exons) } & \multicolumn{5}{|c|}{ Deletions } & \multicolumn{6}{|c|}{ Duplications } & \multirow{2}{*}{$\begin{array}{l}\text { Overall } \\
\text { Sensitivity } \\
\text { (weighted }\end{array}$} \\
\hline & 1 & 2 & 3 & 4 & 5 & 1 & 2 & 3 & 4 & 5 & Exon 11-12 & \\
\hline Sensitivity & $88.9 \%$ & $99.2 \%$ & $100 \%$ & $100 \%$ & $100 \%$ & $70.0 \%$ & $93.8 \%$ & $99.3 \%$ & $100 \%$ & $100 \%$ & $100 \%$ & $96.7 \%$ \\
\hline Weights & $26 \%$ & $21 \%$ & $8 \%$ & $15 \%$ & $26 \%$ & $0 \%$ & $4 \%$ & $0 \%$ & $0 \%$ & $0 \%$ & $\mathrm{n} / \mathrm{a}$ & \\
\hline
\end{tabular}

$[38,39]$. Importantly, Additional file 4: Figure S2 shows that the gene-specific genotypes we observed differed from the Polaris and GIAB data (including phased data on GIAB samples; Additional file 4: Figure S2C). In principle, such differences could arise due in part to errors in either dataset, for example through biological contamination, non-specific amplification, non-specific sequence alignment, or technical processing errors by the chosen genotyping software. However, the concordance between orthogonal hybridcapture and LR-PCR assays suggests that the genotypes we report here are correct. Further, as a third orthogonal method, we also genotyped PMS2 and PMS2CL from RNA extracted from 33 of the LR-PCR samples (see methods). The RNA-derived genotypes were concordant with the LR-PCR data (Additional file 5: Figure S3), strongly suggesting that we elucidated correct gene- and pseudogene-specific genotypes. To aid scientific research and clinical development of PMS2 and its role in lynch syndrome, we share the gene- and pseudogene-specific variant information. For patient samples, to share valuable data while being mindful of patient consent and PHI compliance, we provide variant frequencies (Additional file 3: Table S4). For cell lines, we share variant frequencies, as well as BAM and VCF files for the LR-PCR amplicons spanning the last five exons of PMS2 and PMS2CL (Additional file 6: Table S5 and in ENA accession \#PRJEB27948)

\section{Discussion}

Here we show that a reflex workflow starting with short-read NGS and reflexing to LR-PCR and/or MLPA can detect sequence variants in the last five exons of PMS2 with high analytical sensitivity (>99\% for SNVs/ indels; $>96 \%$ for CNVs) and specificity (>99\% for SNVs/ indels/CNVs). In isolation, short-read sequencing would be incapable of attributing variants to PMS2 or PMS2CL, but it is proficient both at resolving SNVs and indels in PMS2 exon 11 and at flagging samples with other variants whose origin requires disambiguation via reflex testing. In addition to presenting and testing a comprehensive and plausible workflow, we resolved and have shared the PMS2- and PMS2CL-specific genotypes and allele frequencies of many hundreds of clinical and cell line samples. Together, the contributions described herein may advance understanding of PMS2 and facilitate routine screening for Lynch syndrome in HCS offerings.
A high reflex rate after short-read NGS testing (e.g., $>10 \%$ ), while acceptable for the accuracy of a patient's report, may exert unmanageable logistical overhead on the testing laboratory. The reflex rate has two components-one biological and one technical-each with different sources and constraints. The biological component serves as the floor of the reflex rate: if the assay had perfect analytical specificity (i.e., zero false positives) and clinical accuracy (i.e., correct classifications with no VUSs), then there would nevertheless be a nonzero reflex rate due to the presence of pathogenic variants in PMS2 exons $12-15$ and the corresponding PMS2CL regions that need disambiguation. This biological component would, therefore, reflect primarily the integrated population frequency of pathogenic variants across the ambiguous region. The technical component of the reflex rate, by contrast, arises from imperfect analytical specificity and incomplete knowledge of variant pathogenicity. Though higher in our study (99.7\%), analytical specificity for CNVs was $93.7 \%$ in Herman et al. [26], meaning that the technical component of the reflex rate in that study was at least $6.3 \%$ (highlighting the variable nature of the technical component). Also, technical reflex due to VUSs in our workflow was required in $4 \%$ of samples, a share that is expected to drop with further screening of PMS2 and the resulting ability to reclassify VUSs.

There are several laboratory strategies that can yield accurate results for the last five exons of PMS2, though each requires quality-control monitoring. These approaches include LR-PCR with Sanger sequencing, LR-PCR with NGS, MLPA (often requires LR-PCR with sequencing to disambiguate if $\mathrm{CNV}$ in gene or pseudogene), and a reflex workflow built upon short-read NGS as presented here. A risk of performing LR-PCR alone on all samples is allelic dropout, where one of the alleles amplifies poorly or not at all (e.g., due to a SNV under the LR-PCR primer). Appropriate quality control to mitigate this risk could include examining allele balance at sites across the amplicon: allelic dropout is likely if no heterozygous sites are observed, or if all such sites have allele balance significantly less than $50 \%$. We observed one such sample in our cohort with allele balance of $\sim 7 \%$ at SNVs across the amplicon (note that NGS but likely not Sanger sequencing would be able to identify these low-allele-balance SNVs that may be below the Sanger sequencing detection limit); inspection of the hybrid-capture data revealed a SNV under the PMS2 exon-10 LR-PCR 
primer (PMS2_LRPCR_F in Additional file 2: Table S3), which we broadly observed in $0.13 \%$ of patients (i.e., 1 in 769). A shortcoming of monitoring allele balance to flag allelic dropout is that a sample that simply lacks SNVs in the amplified portion of the genome could undergo extensive follow-up characterization without actually being spurious. Ultimately, an asset of a workflow incorporating multiple orthogonal methods-e.g., both LR-PCR and hybrid-capture-based NGS (in parallel or in a reflex arrangement)-is that the different datasets facilitate reconciliation of complicated genotypes.

We attempted to mitigate several potential limitations of our proposed workflow. For instance, our short-read NGS approach in PMS2 exons 12-15 operates variant-calling software with the assumption of a tetraploid background, obviously unusual in a human clinical genomics setting (GATK supports tetraploid calling, but reports of its efficacy in this mode are scarce). Importantly, there was high concordance between short-read-generated SNV calls in the four-copy background and the combined genotypes detected using gene- and pseudogene-specific LR-PCR. A dearth of cell-line or patient samples with indels or CNVs in exons $12-15$ also complicated the ability to assess performance of detecting these important variants. In silico simulations enabled generation of 940 indel-positive and 2186 CNV-positive samples in a tetraploid background, and variant calling on these simulated samples revealed high sensitivity. Finally, despite examination of the workflow's variant-calling accuracy on hundreds of samples, the assay would still require validation before being demonstrated suitable for clinical use.

Although we measured the sensitivity and specificity of the proposed workflow, its potential impact on cost and turnaround time (TAT) of the test were not explored here. The impact on cost and TAT depends greatly on how a laboratory decides to implement the reflex-testing workflow. For instance, TAT could be minimized by running the LR-PCR reactions as soon as samples arrive and then only perform NGS of the amplicons upon flagging by the short-read NGS analysis. But, this approach would incur the cost of generating amplicons in $>90 \%$ of samples that would not need further testing. By contrast, cost is minimized by only doing LR-PCR with NGS on relevant samples after the short-read NGS step, but this approach could increase TAT for those samples. These considerations are ultimately important because TAT and cost impact the utility and accessibility of HCS. It will be exciting to see if future technical developments enable targeted long-read sequencing, as this advance would facilitate clinical-grade testing of highly homologous regions of the genome.

\section{Conclusions}

Screening for pathogenic variants in the last five exons of PMS2 is technically challenging. High homology between PMS2 and PMS2CL complicates identification of gene-specific variants with short-read NGS alone, and reference cell lines that typically aid assay development and validation are not accurately genotyped in public databases. To help overcome these limitations, we have characterized a reflex workflow that achieves high accuracy and publicly shared the gene- and pseudogene-specific raw data, genotypes, and variant frequencies in widely available cell lines.

\section{Additional files}

\begin{abstract}
Additional file 1: Figure S1. Orthogonal datasets used to build the assay. Diagram demonstrating the assays, datasets, algorithms, and analyses used to build the hybrid capture assay for the last five exons of PMS2. The Coriell samples (1b) can be used by other researchers without repeating the LR-PCR as we have made those data publicly available (accession \#PRJEB27948, see Declarations). Genomic DNA (gDNA). (PNG 219 kb)
\end{abstract}

Additional file 2: Table S1. Samples and cell lines used in particular assays and analyses. Table S2. Cell-line samples included in study. Table S3. Oligos and primers used for LR-PCR, RT-PCR, Tn5 adapters. Table S6. Simulated CNV Sensitivity in Cell Line Samples. (XLSX 16 kb)

Additional file 3: Table S4. Allele frequencies from 707 LR-PCRs (XLSX $490 \mathrm{~kb}$ )

Additional file 4: Figure S2. PMS2 exons 11-15 reference genotypes (from Polaris and GIAB) are inconsistent with PMS2 LR-PCR. (A) Concordance between LR-PCR variant calls and Polaris variant calls. (B) Concordance between LR-PCR variant calls and the GIAB multisample call set (including high confidence and filtered variant calls) for all five GIAB samples. (C) Concordance between LR-PCR variant calls and the 10X Genomics haplotype call set available for four GIAB samples. (PNG 76 kb)

Additional file 5: Figure S3. RNA data corroborate hybrid capture and LR-PCR data. (A) Concordance between hybrid capture data and RT-PCR (RNA) for PMS2 and PMS2CL. (B) Concordance between hybrid capture data and LR-PCR (DNA) for PMS2 and PMS2CL. (PNG $135 \mathrm{~kb}$ )

Additional file 6: Table S5. Allele frequencies from $155 \mathrm{GIAB}$ and Polaris LR-PCRs (XLSX 233 kb)

\section{Abbreviations}

CNV: Copy-number variant; FN: False negative; FP: False positive; GIAB: Genome in a Bottle; HCS: Hereditary cancer screening; indels: Short insertions and deletions; LR-PCR: Long-range PCR; MLPA: Multiplex ligation-dependent probe amplification; NEB: New England Biolabs; NGS: Next-generation sequencing; RT-PCR: Reverse transcription PCR; SNV: Single-nucleotide variant; TAT: Turn around time; TN: True negative; TP: True positive; VUS: Variant of uncertain significance

\section{Acknowledgements}

We are grateful to Kaylene Ready, Kristin Price, Leslie Bucheit, Peter Kang, Kevin Haas, Kevin lori, Eric Evans, Krista Moyer, Becca Mar-Heyming, Kerri Hensley, Megan Judkins, Christine Lo, Eric Olson, Kyle Beauchamp, Kristjan Kaseniit, Jeffrey Tratner, Henry Lai, Carly Paul, Pranav Sharma, Victoria Brewster, Irina Ridley, Harris Naemi, and Gabor Brasnjo for support of this manuscript. We thank Invitae for providing CNV-positive samples.

\section{Funding}

Counsyl provided support for the design of the study, data collection, data analysis, data interpretation, and writing.

Availability of data and materials

BAMs for the cell-line LR-PCR data can be found in the European Nucleotide Archive (accession \#PRJEB27948). Corresponding VCFs of these samples are included as a supplemental file. 


\section{Authors' contributions}

GMG, PVG, MRT, DHH, CSC, JRM, GJH, and DM designed the study. GMG, PVG, MRT, LS, IEW, LMM, and RGC collected and analyzed the data. GMG, PVG, MRT, LS, IEW, LMM, GJH, and DM wrote the manuscript. All authors read and approved the final manuscript.

\section{Ethics approval and consent to participate}

The protocol for this study was reviewed and designated as exempt by Western Institutional Review Board.

\section{Consent for publication}

Not applicable.

\section{Competing interests}

All authors are current or former employees and equity holders of Counsyl.

\section{Publisher's Note}

Springer Nature remains neutral with regard to jurisdictional claims in published maps and institutional affiliations.

\section{Received: 31 July 2018 Accepted: 20 September 2018}

\section{Published online: 29 September 2018}

\section{References}

1. Nagy R, Sweet K, Eng C. Highly penetrant hereditary cancer syndromes. Oncogene. 2004;23:6445-70.

2. Lu KH, Wood ME, Daniels M, Burke C, Ford J, Kauff ND, et al. American Society of Clinical Oncology expert statement: collection and use of a cancer family history for oncology providers. J Clin Oncol. 2014;32:833-40.

3. Mucci LA, Hjelmborg JB, Harris JR, Czene K, Havelick DJ, Scheike T, et al. Familial risk and heritability of Cancer among twins in Nordic countries. JAMA. 2016;315:68-76.

4. Foulkes WD. Inherited susceptibility to common cancers. N Engl J Med. 2008;359:2143-53.

5. Garber JE, Offit K. Hereditary cancer predisposition syndromes. J Clin Oncol. 2005;23:276-92

6. Vogelstein B, Papadopoulos N, Velculescu VE, Zhou S, Diaz LA, Kinzler KW. Cancer genome landscapes. Science. 2013;339:1546-58.

7. Vysotskaia VS, Hogan GJ, Gould GM, Wang X, Robertson AD, Haas KR, et al, Development and validation of a 36-gene sequencing assay for hereditary cancer risk assessment. PeerJ. 2017;5:e3046.

8. Kang HP, Maguire JR, Chu CS, Haque IS, Lai H, Mar-Heyming R, et al. Design and validation of a next generation sequencing assay for hereditary BRCA1 and BRCA2 mutation testing. PeerJ. 2016;4:e2162

9. Bunnell AE, Garby CA, Pearson EJ, Walker SA, Panos LE, Blum JL. The clinical utility of next generation sequencing results in a community-based hereditary Cancer risk program. J Genet Couns. 2017;26:105-12.

10. Desmond A, Kurian AW, Gabree M, Mills MA, Anderson MJ, Kobayashi Y, et al. Clinical Actionability of multigene panel testing for hereditary breast and ovarian Cancer risk assessment. JAMA Oncol. 2015;1:943-51.

11. Lynch HT, Smyrk T, Lynch J, Fitzgibbons R Jr, Lanspa S, McGinn T. Update on the differential diagnosis, surveillance and management of hereditary non-polyposis colorectal cancer. Eur J Cancer. 1995;31A:1039-46.

12. Blount J, Prakash $A$. The changing landscape of lynch syndrome due to PMS2 mutations. Clin Genet. 2018:94:61-9.

13. Sijmons RH, RMW H. Review: Clinical aspects of hereditary DNA mismatch repair gene mutations. DNA Repair. 2016;38:155-62.

14. Tiwari AK, Roy HK, Lynch HT. Lynch syndrome in the 21st century: clinical perspectives. QJM. 2016;109:151-8.

15. Lynch HT, Fusaro RM, Lynch JF. Cancer genetics in the new era of molecular biology. Ann N Y Acad Sci. 1997:833:1-28.

16. De Vos M, Hayward BE, Picton S, Sheridan E, Bonthron DT. Novel PMS2 pseudogenes can conceal recessive mutations causing a distinctive childhood cancer syndrome. Am J Hum Genet. 2004;74:954-64.

17. Hayward BE, De Vos M, Valleley EMA, Charlton RS, Taylor GR, Sheridan E, et al. Extensive gene conversion at the PMS2 DNA mismatch repair locus. Hum Mutat. 2007;28:424-30.

18. van der Klift HM, Tops CMJ, Bik EC, Boogaard MW, Borgstein A-M, Hansson KBM, et al. Quantification of sequence exchange events between PMS2 and PMS2CL provides a basis for improved mutation scanning of lynch syndrome patients. Hum Mutat. 2010;31:578-87.
19. Vaughn CP, Robles J, Swensen JJ, Miller CE, Lyon E, Mao R, et al. Clinical analysis of PMS2: mutation detection and avoidance of pseudogenes. Hum Mutat. 2010;31:588-93.

20. Vaughn CP, Hart KJ, Samowitz WS, Swensen JJ. Avoidance of pseudogene interference in the detection of $3^{\prime}$ deletions in PMS2. Hum Mutat. 2011;32: 1063-71.

21. van der Klift HM, Mensenkamp AR, Drost M, Bik EC, Vos YJ, Gille HJJP, et al. Comprehensive mutation analysis of PMS2 in a large cohort of Probands suspected of lynch syndrome or constitutional mismatch repair deficiency syndrome. Hum Mutat. 2016;37:1162-79.

22. Li J, Dai H, Feng Y, Tang J, Chen S, Tian X, et al. A comprehensive strategy for accurate mutation detection of the highly homologous PMS2. J Mol Diagn. 2015;17:545-53.

23. Vaughn CP, Baker CL, Samowitz WS, Swensen JJ. The frequency of previously undetectable deletions involving 3' exons of the PMS2 gene. Genes Chromosomes Cancer. 2013;52:107-12.

24. Espenschied CR, LaDuca H, Li S, McFarland R, Gau C-L, Hampel H. Multigene panel testing provides a new perspective on lynch syndrome. J Clin Oncol. 2017;35:2568-75.

25. Etzler J, Peyrl A, Zatkova A, Schildhaus H-U, Ficek A, Merkelbach-Bruse S, et al, RNA-based mutation analysis identifies an unusual MSH6 splicing defect and circumvents PMS2 pseudogene interference. Hum Mutat. 2008:29:299-305.

26. Herman DS, Smith C, Liu C, Vaughn CP, Palaniappan S, Pritchard CC, et al. Efficient detection of copy number mutations in PMS2 exons with a close homolog. J Mol Diagn. 2018;20:512-21.

27. Li H. Aligning sequence reads, clone sequences and assembly contigs with BWA-MEM [internet]. 2013. Available: http://arxiv.org/abs/1303.3997

28. Li H, Handsaker B, Wysoker A, Fennell T, Ruan J, Homer N, et al. The sequence alignment/map format and SAMtools. Bioinformatics. 2009;25:2078-9.

29. Poplin R, Ruano-Rubio V, DePristo MA, Fennell TJ, Carneiro MO, Van der Auwera GA, et al. Scaling accurate genetic variant discovery to tens of thousands of samples [internet]; 2017. https://doi.org/10.1101/201178.

30. Garrison E, Marth G. Haplotype-based variant detection from short-read sequencing [Internet]. arXiv [q-bio.GN]. 2012. Available: http://arxiv.org/abs/ 1207.3907

31. McKenna A, Hanna M, Banks E, Sivachenko A, Cibulskis K, Kernytsky A, et al. The genome analysis toolkit: a MapReduce framework for analyzing nextgeneration DNA sequencing data. Genome Res. 2010;20:1297-303.

32. Home | Integrative Genomics Viewer [Internet]. [cited 7 Sep 2018]. Available: http://www.broadinstitute.org/igv.

33. Hogan GJ, Vysotskaia VS, Beauchamp KA, Seisenberger S, Grauman PV, Haas $K R$, et al. Validation of an expanded carrier screen that optimizes sensitivity via full-exon sequencing and panel-wide copy number variant identification. Clin Chem. 2018:64:1063-73.

34. Richards S, Aziz N, Bale S, Bick D, Das S, Gastier-Foster J, et al. Standards and guidelines for the interpretation of sequence variants: a joint consensus recommendation of the American College of Medical Genetics and Genomics and the Association for Molecular Pathology. Genet Med. 2015; 17:405-24.

35. Salvatier J, Wiecki TV, Fonnesbeck C. Probabilistic programming in Python using PyMC3. PeerJ Comput Sci. PeerJ Inc. 2016;2:e55.

36. Dobin A, Davis CA, Schlesinger F, Drenkow J, Zaleski C, Jha S, et al. STAR: ultrafast universal RNA-seq aligner. Bioinformatics. 2013;29:15-21.

37. Clopper CJ, Pearson ES. The use of confidence or Fiducial limits illustrated in the case of the binomial. Biometrika. 1934;26:404

38. Zook JM, Catoe D, McDaniel J, Vang L, Spies N, Sidow A, et al. Extensive sequencing of seven human genomes to characterize benchmark reference materials. Sci Data. 2016;3:160025.

39. Zook JM, Chapman B, Wang J, Mittelman D, Hofmann O, Hide W, et al. Integrating human sequence data sets provides a resource of benchmark SNP and indel genotype calls. Nat Biotechnol. 2014;32:246-51. 